\title{
New guidelines for evaluating health tech
}

Cite as: CMAJ 2017 May 8;189:E675. doi: 10.1503/cmaj.1095415

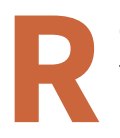

esearchers looking to consistently translate health data into an economic analysis that decisionmakers can actually use will want to refer to the updated guidelines from the Canadian Agency for Drugs and Technologies in Health (CADTH).

"The guidelines are best-practice standards," says Karen Lee, director of health economics for CADTH, a not-for-profit funded by federal and provincial governments. "They help researchers to have a standardized approach."

The fourth edition of the Guidelines for the Economic Evaluation of Health Technologies: Canada, provides essential information for those making decisions about the most efficacious pharmaceuticals, devices and methods of treating and diagnosing Canadians. It sets out how to effectively and consistently determine the economic value of health technologies compared to existing approaches.

The revised document brings two major changes. It emphasizes the need for researchers to focus on the problem they are investigating to ensure it is relevant in the broader health system context. Economic analysis is not an academic exercise, says Lee. "Researchers really need to understand the problem from the decision-maker's perspective. Researchers need to have those conversations."

The new 76-page edition also calls on researchers to use a reference case, a set of recommended methods that promote uniformity and transparency. It allows dif-

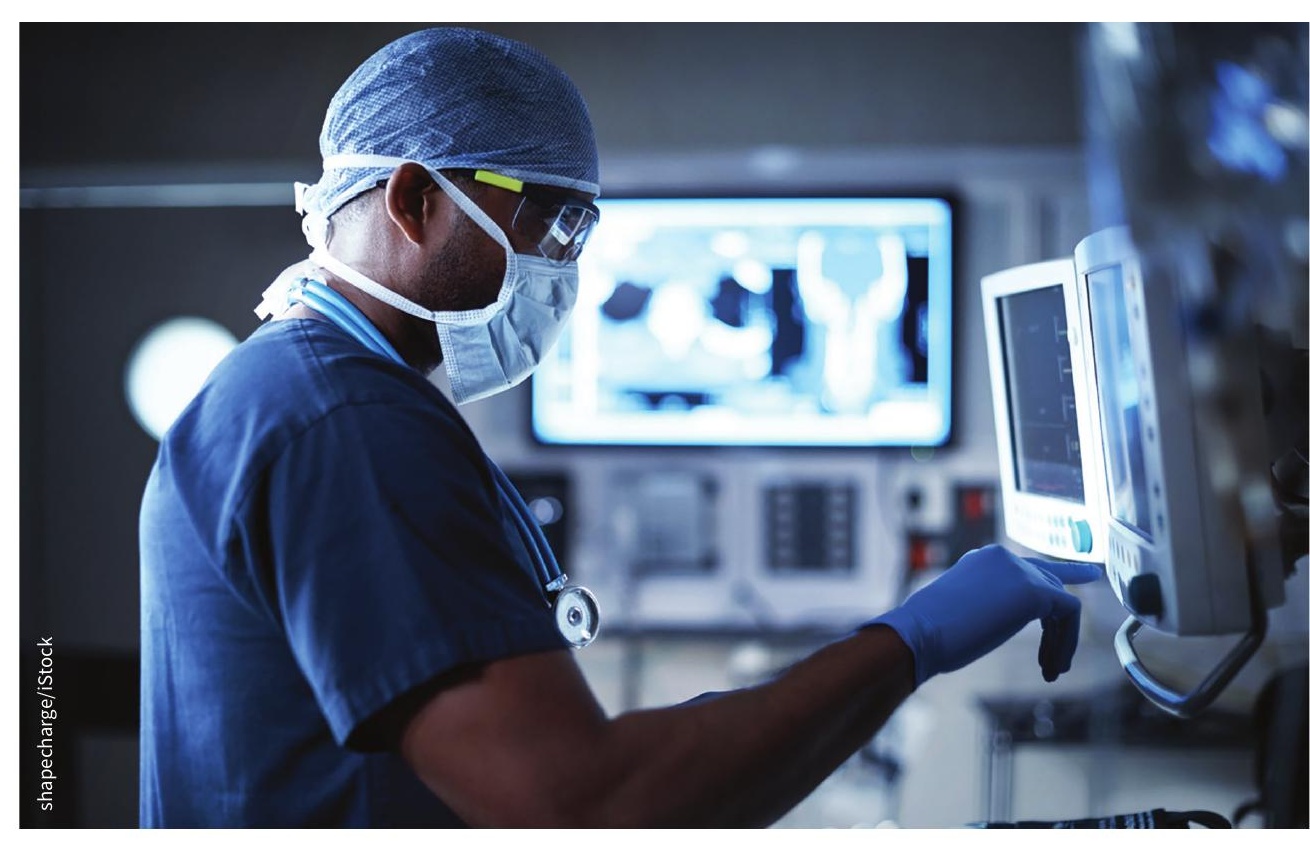

New guide to economic analysis of drugs and technology.

ferent technologies and decisions to be effectively compared. An appendix in the new guidelines lays out the components of a reference case, including identifying the populations for which interventions are to be used.

Advances in technology are also reflected in the guidelines, which were first published in 1994 and last updated in 2006. In that edition, a probabilistic analysis, which provides a more realistic sense about the accuracy of a cost-effectiveness estimate, was not recommended as a standard measure because of the computing time involved. "Today this type of analysis can be done in a couple of hours or less. That leads to greater certainty in the results," says Lee.

CADTH is already planning for the next round of changes to the guidelines, which are downloaded 12000 times a year and used internationally as a model to evaluate new health technologies. Emerging areas being watched closely include the use of real-world data from provincial health databases and new forms of clinical trials, such as adaptive clinical trials.

"It's a bit of a living document," says Lee.

donalee Moulton, Halifax 